University of Nebraska - Lincoln

DigitalCommons@University of Nebraska - Lincoln

Faculty Papers and Publications in Animal

Science

Animal Science Department

January 2004

\title{
A large-sample QTL study in mice: I. Growth
}

Joao L. Rocha

University of Nebraska-Lincoln

Eugene J. Eisen

North Carolina State University, Raleigh, North Carolina

L. Dale Van Vleck

University of Nebraska-Lincoln, dvan-vleck1@unl.edu

Daniel Pomp

University of Nebraska-Lincoln, dpomp1@unl.edu

Follow this and additional works at: https://digitalcommons.unl.edu/animalscifacpub

Part of the Animal Sciences Commons

Rocha, Joao L.; Eisen, Eugene J.; Van Vleck, L. Dale; and Pomp, Daniel, "A large-sample QTL study in mice: I. Growth" (2004). Faculty Papers and Publications in Animal Science. 234.

https://digitalcommons.unl.edu/animalscifacpub/234

This Article is brought to you for free and open access by the Animal Science Department at DigitalCommons@University of Nebraska - Lincoln. It has been accepted for inclusion in Faculty Papers and Publications in Animal Science by an authorized administrator of DigitalCommons@University of Nebraska - Lincoln. 


\title{
Mammälian G
}

\section{A large-sample QTL study in mice: I. Growth}

\author{
Joao L. Rocha, ${ }^{1, \star}{ }^{\star}$ Eugene J. Eisen, ${ }^{2}$ L. Dale Van Vleck, ${ }^{3}$ Daniel Pomp ${ }^{1}$ \\ ${ }^{1}$ Department of Animal Science, University of Nebraska, Lincoln, Nebraska 68583-0908, USA \\ ${ }^{2}$ Department of Animal Science, North Carolina State University, Raleigh, North Carolina, 27695-7621, USA \\ ${ }^{3}$ USDA, ARS, USMARC, Lincoln, Nebraska 68583-0908, USA
}

Received: 8 July 2003 / Accepted: 10 September 2003

\begin{abstract}
By use of long-term selection lines for high and low growth, a large-sample $\left(n=\sim 1,000 \mathrm{~F}_{2}\right)$ experiment was conducted in mice to further understand the genetic architecture of complex polygenic traits. In combination with previous work, we conclude that QTL analysis has reinforced classic polygenic paradigms put in place prior to molecular analysis. Composite interval mapping revealed large numbers of QTL for growth traits with an exponential distribution of magnitudes of effects and validated theoretical expectations regarding gene action. Of particular significance, large effects were detected on Chromosome (Chr) 2. Regions on Chrs 1, 3, 6, 10, 11, and 17 also harbor loci with significant contributions to phenotypic variation for growth. Despite the large sample size, average confidence intervals of $\sim 20 \mathrm{cM}$ exhibit the poor resolution for initial estimates of QTL location. Analysis with genome-wide and chromosomal polygenic models revealed that, under certain assumptions, large fractions of the genome may contribute little to phenotypic variation for growth. Only a few epistatic interactions among detected QTL, little statistical support for gender-specific QTL, and significant age effects on genetic architecture were other primary observations from this study.
\end{abstract}

The last 15 years have witnessed a strong impetus towards the dissection of the genetic architecture of complex traits in livestock (e.g., reviews by Andersson 1998; Georges 1998); plants (e.g., Frary et al. 2000; Yano 2001); laboratory species (e.g., Pomp 1997; Moore and Nagle 2000; Mackay 2001); and humans (e.g., Weiss 1996; Terwilliger and Goring

${ }^{\star}$ Present address: Sygen International, 2929 Seventh Street, Berkeley, California 94710, USA

Correspondence to: D. Pomp; E-mail: dpomp@unl.edu
2000). An era introduced by Alan Robertson (Robertson 1966), and made possible by developments at the molecular and analytical levels (e.g., Botstein et al. 1980; Mullis et al. 1992), is rapidly coming to fruition in general lessons that can be inferred from the depth and variety of exploratory QTL studies.

As inadequate sample sizes have been a characteristic of many preliminary QTL studies (Beavis 1998; Young 1999|, a large-sample QTL study was undertaken in mice that could help refine our general understanding of the genetic architecture underlying quantitative phenotypic variation observed in segregating populations of experimental crosses. Results for growth traits are reported here; results for body composition (organ weights and adiposity) in males and reproduction (ovulation rate, litter size, embryonic survival) in females are presented in companion papers (Rocha et al. 2003b, 2003c). Primary objectives of this study were to examine general concepts of quantitative genetics theory regarding numbers and magnitudes of polygenic effects, the nature of polygene action, and the importance of epistatic interactions. Other largesample QTL analyses of growth have been conducted by Cheverud et al. (1996) and Vaughn et al. (1999), and their results can be joined with those of the present study to draw general conclusions regarding the underlying nature of quantitative phenotypic variation.

\section{Materials and methods}

Mouse lines and crosses. Two lines of mice selected for increased 3- to 6-week weight gain (M16i; Hanrahan et al. 1973) and low 6-week weight (L6; Legates 1969) were intercrossed to produce an $F_{2}$ generation $(\mathrm{n}=990)$. Line M16i, derived from an outbred population (ICR), underwent within full-sib family selection for 27 generations for high weight gain from 21 to 42 days. Subsequently, 18 generations of full-sib mating with minimal selection were 
implemented to produce the inbred line used in this study. Line L6, derived from a cross of four inbred lines (A/J, BALB/C, DBA/2J, and AKR/J), underwent within-family selection for low 6-week body weight for 25 generations, followed by 128 generations of random selection. The cumulative inbreeding coefficient of L6 at the time of this study was estimated to be greater than 0.9 .

To study the extent of phenotypic divergence between these two lines, mice were produced with standardized pre-weaning fraternal sizes of six. Least-squares estimates for 3-, 6-, and 10-week body weights for the M16i and L6 lines were, respectively, 16.6 vs. 7.7 g, 41.5 vs. 14.8 g, and 57.9 vs. 18.4 g. Feed intake during the 3 - to 6 -week period was significantly lower in L6 in proportion to their smaller body size ( $2.9 \mathrm{~g}$ /day vs. $6.8 \mathrm{~g} /$ day for M16i).

To generate the $\mathrm{F}_{2}$ cross, L6 males were mated to M16i females, with the resulting $F_{1} s$ inter se mated (no full-sib pairings) in two consecutive replicates encompassing a total of 64 full-sib $\mathrm{F}_{2}$ families. The $\mathrm{F}_{2}$ litters were standardized one day after birth to 10 pups. Mice were reared at $21^{\circ} \mathrm{C}$ and $55 \%$ relative humidity and in a 12:12 h light:dark cycle. Food and water were provided ad libitum. Purina Mouse Chow 5015 was available from mating through weaning of pups at 3 weeks, and Purina Laboratory Chow 5001 was provided subsequent to weaning. Body weight was recorded on all $\mathrm{F}_{2}$ mice at 21,42 , and 70 days of age. Tail length was measured at day 70 as an indicator of skeletal growth.

Genotyping. Sixty-three fully informative microsatellite markers spanning the 19 autosomes were genotyped in the complete $F_{2}$ population. Genotypes were determined by standard PCR and agarose gel electrophoresis protocols. In addition, a dominant PCR-based marker at the Ped locus (Green 1989; $\mathrm{Xu}$ et al. 1994) was genotyped in $\mathrm{F}_{2}$ females ( $n$ $=439$ ) the scoring of a band for this marker indicated the presence of either one or two M16i alleles. All genotypes were scored twice, and discrepancies were rectified. Marker $\mathrm{F}_{2}$ segregation ratios were evaluated for deviations from Mendelian expectations by chi-square test (Ott 1984), and the GENOTYPES and ERROR DETECTION features of MAPMAKER/EXP (Lincoln and Lander 1992; Lincoln et al. 1992) were implemented to detect and correct possible anomalies.

Marker linkage maps were generated with MAPMAKER/EXP (Lincoln et al. 1992), and estimated marker distances (Fig. 1; Kosambi cM) were used for QTL analyses. Reasonable agreement was found with the Mouse Genome Database (MGD) maps, although this would have been enhanced with the Carter-Falconer mapping function (Carter and Falconer 1951). Although a denser map would be desirable in a few genomic regions, marker coverage in this study is as prescribed for initial marker-QTL linkage experiments (Darvasi and Soller 1994).

Data analyses. Nine quantitative traits were analyzed for location and magnitude of underlying QTL: 3-week weight (WT3wk), 6-week weight (WT6wk), 10-week weight (WT10wk), tail length (TAIL), growth between weeks 3 and 6 (EARLYGR), growth between weeks 6 and 10 (LATEGR), EARLYGR adjusted for WT3wk (EARLADJ), LATEGR adjusted for WT6wk (LATEADJ), and tail length adjusted for 6-week body wt (TAILADJ). Adjustments of growth rates represented attempts to detect QTL possibly involved in expression of time-specific episodes of new genetic variation for growth (Atchley and Zhu 1997). Although measured at 10 weeks of age, the correlation of tail length with WT6wk was considerably stronger than that with WT10wk. Means and basic statistics for all traits are in Table 1 and phenotypic correlations are in Table 2.

The most appropriate statistical model was identified for each trait by fitting generalized linear models (PROC GLM; SAS Institute Inc. 1985,1996), including fixed effects of sex, replicate/parity, fullsib family/litter, and respective interactions, if significant. Residuals were analyzed with QTL Cartographer (Version 1.15; Basten et al. 2001) to perform composite interval mapping (CIM; Zeng 1993, 1994). A forward-backward stepwise regression procedure with a 0.001 threshold for the addition and elimination of new markers was used to select background factors, and a $10-\mathrm{cM}$ window size was adopted. Genome-wise significance thresholds were established with at least 1,000 permutations (Churchill and Doerge 1994). Initially, 100 permutations were conducted for each of the nine traits, and when similar results were observed for all traits, their permutations were pooled, leading to adoption of LOD $=3.3$ for the $5 \%$ genomewise significance threshold. One LOD-drop support intervals (Lander and Botstein 1989) were considered acceptable approximations for 90-95\% confidence intervals of QTL location (Van Ooijen 1992). The models used and assumptions made were found to be adequate based on residual analysis and model diagnostics (Hackett 1997).

Marker-based GLM models were used to test all pair-wise and higher-order epistatic interactions among markers included in the final selected models (Brockmann et al. 2000), and also to test all interactions between these markers and the fixed effects. To examine the possibility of marker $\mathrm{X}$ sex interac- 


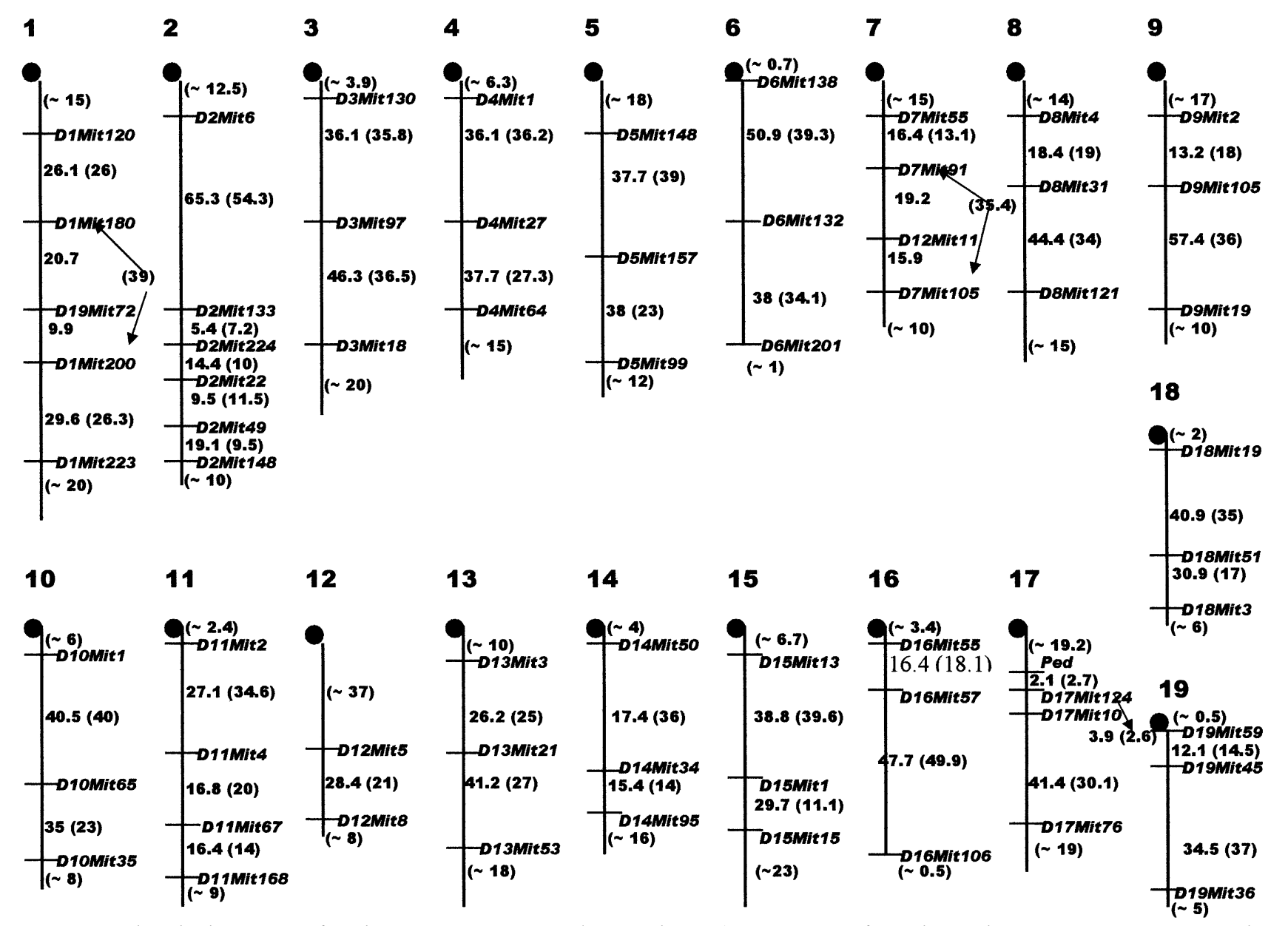

Fig. 1. Marker linkage-map for the M16i $\times$ L6 cross (Kosambi cM). Positions of markers closest to centromere and total chromosome lengths are from the Mouse Genome Database (MGD, May 2002). Comparative MGD inter-marker distances are in parentheses. Arrows on Chr 1 and 7 pertain to situations where two markers have mapped to chromosomes other than those expected based on MGD.

tions, separate CIM analyses were conducted for each gender. The proportion of within-family residual phenotypic variation explained by each QTL was determined as the coefficient of determination $\left(\mathrm{R}^{2}\right)$.

"Genome substitution effects" and "chromosomal polygenic" models (Visscher and Haley 1996; Knott et al. 1998; Schork 2001) were used to distin-

Table 1. Basic statistics for growth traits in the M16i $\times$ L6 F2 cross

\begin{tabular}{lcrlr}
\hline \multicolumn{1}{c}{ Trait $^{\mathrm{a}}$} & $N$ & Mean & Std. Dev. & Range \\
\hline Wt3wk (g) & 990 & 13.4 & 1.9 & $7.0-21.5$ \\
Wt6wk (g) & 990 & 27.2 & 3.9 & $13.1-42.4$ \\
Wt10wk (g) & 989 & 32.4 & 5.3 & $18.3-49.1$ \\
TAIL (cm) & 990 & 7.5 & 0.48 & $5.1-9.1$ \\
EARLYGR (g) & 987 & 13.8 & 3.3 & $2.9-28.1$ \\
LATEGR (g) & 986 & 5.2 & 3.3 & $-2.5-21.4$
\end{tabular}

a-week weight (WT3wk), 6-week weight (WT6wk), 10-week weight (WT10wk), tail length (TAIL), growth between weeks 3 and 6 (EARLYGR), and growth between weeks 6 and 10 (LATEGR). guish between single QTL with large effect and multiple linked QTL. Marker-genotypes were assigned additive scores of $1,0.5$, or 0 , according to whether they were homozygous for the M16i allele, heterozygous, or homozygous for the $L 6$ allele. For each $F_{2}$ individual, scores were summed over all 64 markers (genome substitution effect model), or on a chromosomal basis (chromosomal polygenic models), and fitted as a covariate in the context of the best statistical model previously identified for each trait. The same was done to compute genome- or chromosome-level dominance scores, except that heterozygotes were assigned a value of 1 , while both homozygotes were assigned values of 0 . Unequal marker spacing issues were ignored.

Coefficients of determination from the genome substitution model ( $2 \mathrm{df} /$ genome) were contrasted with those from aggregate chromosomal polygenic models (2 df/chromosome or $2 \mathrm{df} /$ subset of the genome) including only chromosomes where QTL had been detected, and finally with the $\mathrm{R}^{2}$ from models 
Table 2. Phenotypic correlations ${ }^{\mathrm{a}}$ (top row) and QTL congruencies ${ }^{\mathrm{b}}$ (bottom row) among growth traits

\begin{tabular}{|c|c|c|c|c|c|c|}
\hline Trait $^{\mathrm{c}}$ & WT3wk & WT6wk & WT10wk & TAIL & $E A R L Y G R$ & LATEGR \\
\hline Wt3wk & 1.0 & 0.56 & 0.47 & 0.53 & 0.10 & 0.09 \\
\hline & 1.0 & 0.42 & 0.40 & 0.15 & 0.32 & 0.15 \\
\hline Wt6wk & - & 1.0 & 0.79 & 0.51 & 0.88 & 0.09 \\
\hline & & 1.0 & 0.67 & 0.15 & 0.71 & 0.15 \\
\hline Wt10wk & - & - & 1.0 & 0.36 & 0.69 & 0.68 \\
\hline & & & 1.0 & 0.14 & 0.50 & 0.33 \\
\hline TAIL & - & - & - & 1.0 & 0.31 & $-0.01^{\text {n.s. }}$ \\
\hline EARLYGR & - & - & - & $\begin{array}{l}1.0 \\
-\end{array}$ & $\begin{array}{l}0.06 \\
1.0 \\
1.0\end{array}$ & $\begin{array}{l}0.20 \\
0.06^{\text {n.s. }} \\
0.13\end{array}$ \\
\hline
\end{tabular}

${ }^{\mathrm{a}}$ Most correlations highly significant $(P<0.0001)$. ${ }^{\text {n.s. }}$ indicates $P>0.05$.

${ }^{\mathrm{b}} \mathrm{QTL}$ common to both traits (overlapping confidence intervals) as a fraction of the total number of QTL detected for the two traits.

c3-week weight (WT3wk), 6-week weight (WT6wk), 10-week weight (WT10wk), tail length (TAIL), growth between weeks 3 and 6 (EARLYGR) and growth between weeks 6 and 10 (LATEGR).

including only those significant markers that had previously been selected with the marker-based GLM models (2 df/marker or $2 \mathrm{df} /$ marker subset). These analyses assume that all quantitative trait alleles contributed by a particular parental line are in cis alignment for each of the traits tested (i.e., all M16i alleles are assumed to either increase or decrease the particular trait), which was true for the growth traits in this study. Quadratic effects of these genomic and chromosomal covariates were found to not be statistically significant.

\section{Results}

Deviations from Mendelian segregation ratios. A few markers deviated considerably from Mendelian $\mathrm{F}_{2}$ segregation ratios (chi-square test; $P<0.001$ ). No reason to exclude them from the study could be found, and true segregation distortion was assumed. Marker D18Mit3 had a considerable deficit of M16i homozygotes and considerable excess of L6 allele homozygotes. A consistent pattern of segregation distortion was also observed for a group of linked markers on Chromosome (Chr) 2. This pattern is discussed in relation to QTL for prenatal fitness in Rocha et al (2003c).

QTL analyses. Results for all QTL identified are presented in Table 3. For weight and growth traits, 89 significant QTL were detected (Table 3). Eighteen QTL were found for WT10wk, 17 each for WT3wk and WT6wk, 12 for EARLYGR, 6 each for LATEGR and TAIL, 4 each for EARLADJ and TAILADJ, and 5 for LATEADJ. QTL were detected on all chromosomes except for MMU14 and 16, for which likelihood plots were remarkably flat. A thorough comparison of QTL detected relative to those found in previous studies is provided in Table 3 . To deter- mine correspondences between map positions of specific QTL, a useful test is available (Keightley and Knott 1999).

Average additive QTL effects expressed in phenotypic standard deviation units (Table 4) and as a percentage of residual variance (Table 5) each had fairly similar patterns for all unadjusted traits, with the low minimum values reflecting the considerable statistical power of this study. However, the large average confidence intervals for QTL locations ( 20 cM; Table 5) illustrate the poor precision of QTL mapping in an $F_{2}$ context even with large sample sizes, although resolution may have been hampered by the relatively sparse marker coverage. Average dominance effects (Tables 4 and 5) were large for early growth traits, but less important for later growth traits. Distributions of estimates of additive and dominance effects are summarized graphically in Figure 2. Very few QTL were found for which a significant dominance effect was identified in the absence of a significant additive effect.

For WT3wk, all M16i alleles were dominant (Tables 5 and 6). A trend towards additivity is seen with increasing maturity, and by 6 weeks L6 alleles become dominant for a considerable fraction of the QTL. This pattern is also true for QTL that map to the same genomic regions and that are likely to be the same QTL impacting different growth periods. Since M16i alleles remain dominant for a nearly equal fraction of the QTL, the resulting aggregate effect is overall additivity for WT6wk and WT10wk, as demonstrated by the average estimates of relative dominance (Table 4). This reversal of dominance and the periods when it happens are further demonstrated by the negative average estimates of relative dominance QTL effects observed for both EARLYGR and LATEGR, indicating that the initial fast early growth of individuals with multiple heterozygous 
Table 3. QTL detected and respective statistics by chromosome

\begin{tabular}{|c|c|c|c|c|c|c|c|c|}
\hline$M M U$ & Symbol & Flanking Markers ${ }^{\mathrm{b}}$ & Position $^{\mathrm{c}}(\mathrm{cM})$ & $a^{\mathrm{d}}$ & $d^{\mathrm{e}}$ & $\% V^{\mathrm{f}}$ & $L O D$ & Other studies ${ }^{\mathrm{g}}$ \\
\hline 1 & $W 3 q 13$ & $120-180$ & $\begin{array}{l}32.9 \\
23.0-41.0\end{array}$ & 0.12 & 1.2 & 1.9 & 4.0 & B3, Ch, Md, Mr, R \\
\hline 1 & $W 3 q 12$ & $180-72$ & $\begin{array}{l}51.3 \\
41.0-67.4\end{array}$ & 0.12 & 0.87 & 1.5 & 4.2 & B3, Ch, Kt, Md, Mr, R, V \\
\hline 1 & $\operatorname{Lgq} 5$ & $180-72$ & $\begin{array}{l}53.7 \\
32.9-70.0\end{array}$ & 0.15 & -0.49 & 2.4 & 3.6 & $\mathrm{~A}, \mathrm{Ch}, \mathrm{Md}, \mathrm{Mr}, \mathrm{R}, \mathrm{V}$ \\
\hline 1 & W10q6 & $180-72$ & $\begin{array}{l}56.3 \\
48.7-66.5\end{array}$ & 0.19 & 0.02 & 3.9 & 9.7 & $\mathrm{~B} 3, \mathrm{Ch}, \mathrm{Kt}, \mathrm{Md}, \mathrm{Mr}, \mathrm{V}$ \\
\hline 1 & W6q14 & $180-72$ & $\begin{array}{l}61.4 \\
46.1-77.6\end{array}$ & 0.14 & 0.34 & 1.5 & 4.1 & A, B3, Ch, H, Kt, Md, Mr, V \\
\hline 1 & $W 10 q 7$ & $72-200$ & $\begin{array}{l}72.6 \\
66.5-79.0\end{array}$ & 0.17 & -0.08 & 3.0 & 9.1 & $\mathrm{~A}, \mathrm{Ch}, \mathrm{H}, \mathrm{Kt}, \mathrm{Md}, \mathrm{V}$ \\
\hline 1 & $\operatorname{Lgq} 4$ & $200-223$ & $\begin{array}{l}92.3 \\
70.0-?\end{array}$ & 0.17 & -0.32 & 2.9 & 3.7 & A, Ch, H, V \\
\hline 1 & $\operatorname{Lgaq3}$ & $200-223$ & $\begin{array}{l}95.9 \\
86.1-?\end{array}$ & 0.19 & -0.72 & 3.2 & 4.5 & Ch, H, V \\
\hline 2 & Egq1 & $6-133$ & $\begin{array}{l}49.1 \\
42.4-60.7\end{array}$ & 0.42 & 0.14 & 13.9 & 12.4 & $\mathrm{~B} 2, \mathrm{C}, \mathrm{Ch}, \mathrm{D}, \mathrm{H}, \mathrm{M}, \mathrm{Ms}, \mathrm{V}$ \\
\hline 2 & W6q1 & $6-133$ & $\begin{array}{l}50.8 \\
45.8-57.5\end{array}$ & 0.56 & 0.23 & 24.2 & 24.3 & $\mathrm{~B} 2, \mathrm{C}, \mathrm{D}, \mathrm{H}, \mathrm{M}, \mathrm{Ms}, \mathrm{V}$ \\
\hline 2 & W10q9 & $6-133$ & 50.8 & 0.34 & 0.25 & 23.6 & 8.6 & B2, C, Ch, D, H, M, Ms, V \\
\hline 2 & W3q1 & $6-133$ & $\begin{array}{l}54.1 \\
47.4-62.4\end{array}$ & 0.42 & 0.76 & 13.2 & 17.9 & $\mathrm{~B} 2, \mathrm{C}, \mathrm{Ch}, \mathrm{D}, \mathrm{H}, \mathrm{M}, \mathrm{Ms}, \mathrm{V}$ \\
\hline 2 & $W 3 q^{2}$ & $224-22$ & $\begin{array}{l}78.2 \\
74.0-82.4\end{array}$ & 0.27 & 0.51 & 3.3 & 12.0 & $\mathrm{C}, \mathrm{Ch}, \mathrm{D}, \mathrm{L}, \mathrm{M}, \mathrm{V}, \mathrm{Y}$ \\
\hline 2 & W6q2 & $224-22$ & $\begin{array}{l}78.2 \\
75.4-81.0\end{array}$ & 0.31 & -0.06 & 5.7 & 17.4 & $\mathrm{C}, \mathrm{Ch}, \mathrm{D}, \mathrm{L}, \mathrm{M}, \mathrm{V}, \mathrm{Y}$ \\
\hline 2 & Egq2 & $224-22$ & $\begin{array}{l}78.2 \\
74.7-81.7\end{array}$ & 0.21 & -0.43 & 3.7 & 9.7 & $\mathrm{C}, \mathrm{Ch}, \mathrm{D}, \mathrm{L}, \mathrm{M}, \mathrm{V}, \mathrm{Y}$ \\
\hline 2 & W10q1 & $224-22$ & $\begin{array}{l}79.6 \\
76.8-81.7\end{array}$ & 0.35 & -0.03 & 9.4 & 29.0 & C, Ch, D, L, M, V, Y \\
\hline 2 & $\operatorname{Lgq2}$ & $224-22$ & $\begin{array}{l}82.4 \\
77.5-92.5 \\
82.4\end{array}$ & 0.18 & -0.16 & 2.2 & 5.5 & C, Ch, D, L, M, P, V, Y \\
\hline 2 & T1q2 & $22-49$ & $\begin{array}{l}82.4 \\
76.8-93.7 \\
91.2\end{array}$ & 0.19 & 0.25 & 1.7 & 7.2 & $C, C 11, D, D, 1+1,1,8,1$ \\
\hline 2 & $T 1 q 4$ & $49-148$ & $\begin{array}{l}83.9-95.5 \\
97.5\end{array}$ & 0.19 & 0.08 & 2.0 & 7.1 & Ch2 \\
\hline 3 & $\operatorname{Lgq3}$ & $130-97$ & $\begin{array}{l}15.8 \\
?-27.7\end{array}$ & 0.18 & -0.33 & 2.5 & 4.5 & $\mathrm{Md}$ \\
\hline 3 & $\operatorname{Lgaq} 4$ & $130-97$ & $\begin{array}{l}19.8 \\
?-33.7\end{array}$ & 0.19 & -0.49 & 2.4 & 3.8 & Ch, Md \\
\hline 3 & W10q3 & $130-97$ & $\begin{array}{l}29.7 \\
23.7-40.5\end{array}$ & 0.26 & -0.09 & 7.0 & 15.5 & Ch \\
\hline 3 & Egq3 & $97-18$ & $\begin{array}{l}39.7 \\
32.7-52.3\end{array}$ & 0.18 & -0.56 & 4.8 & 8.8 & Ch, Md \\
\hline 3 & Egaq-1 & $97-18$ & $\begin{array}{l}39.7 \\
25.7-53.1\end{array}$ & 0.15 & -0.59 & 2.9 & 5.7 & Ch, Md \\
\hline 3 & W6q7 & $97-18$ & $\begin{array}{l}47.7 \\
31.7-56.4\end{array}$ & 0.23 & -0.20 & 7.0 & 9.3 & Ch, Md \\
\hline 3 & W10q4 & $97-18$ & $\begin{array}{l}47.7 \\
40.5-54.0\end{array}$ & 0.26 & -0.03 & 9.5 & 14.9 & Ch, Md \\
\hline 3 & $W 3 q 9$ & $97-18$ & $\begin{array}{l}52.9 \\
41.9-60.8\end{array}$ & 0.18 & 1.2 & 4.2 & 5.5 & Ch, Md \\
\hline 4 & W6q15 & $1-27$ & $\begin{array}{l}36.4 \\
18.4-42.5\end{array}$ & 0.13 & 0.13 & 2.4 & 3.6 & B3, Ch-2, Kt, Lt, Md, Pl, V \\
\hline 4 & W6q13 & $27-64$ & $\begin{array}{l}55.3 \\
43.7-64.0\end{array}$ & 0.18 & 0.38 & 2.4 & 4.8 & B2-3, Ch, K, Lt, Mr, Pl, V \\
\hline
\end{tabular}


Table 3. Continued

\begin{tabular}{|c|c|c|c|c|c|c|c|c|}
\hline$M M U$ & Symbol & Flanking Markers ${ }^{\mathrm{b}}$ & Position $^{\mathrm{c}}(\mathrm{cM})$ & $a^{\mathrm{d}}$ & $d^{\mathrm{e}}$ & $\% \mathrm{~V}^{\mathrm{f}}$ & $L O D$ & Other studies ${ }^{\mathrm{g}}$ \\
\hline 4 & W10q10 & $27-64$ & $\begin{array}{l}55.3 \\
48.1-61.8\end{array}$ & 0.21 & -0.12 & 4.8 & 8.3 & B2-3, Ch, K, Lt, Mr, Pl, V \\
\hline 4 & $W 3 q 17$ & $27-64$ & $\begin{array}{l}63.7 \\
53.6-?\end{array}$ & 0.13 & 0.63 & 1.6 & 3.6 & $\mathrm{~B} 2-3, \mathrm{Ch}, \mathrm{K}, \mathrm{Mr}, \mathrm{Pl}, \mathrm{V}$ \\
\hline 5 & $\operatorname{Lgq} 6$ & $148-157$ & $\begin{array}{l}26.3 \\
?-42.9\end{array}$ & 0.15 & -0.11 & 2.8 & 3.5 & $\mathrm{Kl}$ \\
\hline 5 & $W 10 q 16$ & $148-157$ & $\begin{array}{l}42.8 \\
29.4-61.8\end{array}$ & 0.13 & 0.62 & 2.8 & 4.3 & $\mathrm{~B} 2, \mathrm{Kl}, \mathrm{Kt}$ \\
\hline 6 & Egq8 & $138-132$ & $\begin{array}{l}25.4 \\
16.9-40.0\end{array}$ & 0.22 & 0.19 & 5.3 & 5.2 & $\mathrm{~A}, \mathrm{Ch}-2, \mathrm{Kt}, \mathrm{Ms}, \mathrm{R}$ \\
\hline 6 & W6q4 & $138-132$ & $\begin{array}{l}27.021 .6-36.3 \\
21.6-36.3\end{array}$ & 0.31 & 0.34 & 11.0 & 12.1 & A, Ch-2, Kt, Ms, R \\
\hline 6 & $W 3 q 3$ & $138-132$ & $\begin{array}{l}28.5 \\
20.8-49.0\end{array}$ & 0.26 & 0.33 & 5.5 & 10.1 & $\mathrm{~A}-2, \mathrm{Ch}-2, \mathrm{Kt}, \mathrm{Ms}, \mathrm{R}$ \\
\hline 6 & $W 10 q 11$ & $138-132$ & $\begin{array}{l}28.5 \\
20.0-47.2\end{array}$ & 0.19 & 0.66 & 8.0 & 7.9 & $\mathrm{~A}-2, \mathrm{Ch}-2, \mathrm{Kt}, \mathrm{Ms}, \mathrm{R}$ \\
\hline 6 & W6q5 & $132-201$ & $\begin{array}{l}49.5 \\
39.3-55.8\end{array}$ & 0.26 & 0.35 & 6.3 & 11.3 & A-2, Ch-2, Ms, V \\
\hline 6 & Egq11 & $132-201$ & $\begin{array}{l}51.2 \\
40.0-61.1\end{array}$ & 0.18 & 0.21 & 3.0 & 4.8 & A-2, Ch-2, Ms, V \\
\hline 7 & Tlq5 & $55-91$ & $\begin{array}{l}15.0 \\
?-22.2\end{array}$ & 0.13 & 0.67 & 1.7 & 4.6 & $\mathrm{Mr}$ \\
\hline 7 & W6q17 & $55-91$ & $\begin{array}{l}18.2 \\
?-27.0\end{array}$ & 0.12 & 0.43 & 1.7 & 3.4 & $\mathrm{~B} 3, \mathrm{Ch}, \mathrm{Kt}, \mathrm{Md}, \mathrm{Ms}$ \\
\hline 7 & W10q18 & $55-91$ & $\begin{array}{l}18.2 ?-27.0 \\
?-27.0\end{array}$ & 0.12 & 0.03 & 1.9 & 4.1 & $\mathrm{~B} 3, \mathrm{Ch}, \mathrm{Kt}, \mathrm{Md}, \mathrm{Ms}$ \\
\hline 7 & $W 3 q 10$ & $55-91$ & $\begin{array}{l}24.6 \\
18.2-28.1\end{array}$ & 0.12 & 1.1 & 1.8 & 5.3 & B3, Ch, Kt, Md \\
\hline 7 & $W 3 q 8$ & $91-11$ & $\begin{array}{l}34.2 \\
28.1-42.3\end{array}$ & 0.14 & 1.0 & 1.8 & 5.5 & $\mathrm{~B} 3, \mathrm{Ch}, \mathrm{Kt}, \mathrm{Md}, \mathrm{T}, \mathrm{V}$ \\
\hline 8 & Egq12 & $4-31$ & $\begin{array}{l}24.3 \\
?-42.2\end{array}$ & 0.14 & 0.05 & 1.1 & 3.5 & C, Ch-2, V \\
\hline 8 & W10q14 & $4-31$ & $\begin{array}{l}26.4 \\
19.2-41.4\end{array}$ & 0.13 & 0.50 & 1.3 & 5.2 & C, Ch-2, V \\
\hline 8 & W6q9 & $4-31$ & $\begin{array}{l}28.5 \\
21.2-43.7\end{array}$ & 0.19 & 0.29 & 2.1 & 7.6 & C, Ch-2, V \\
\hline 8 & W3q5 & $31-121$ & $\begin{array}{l}36.6 \\
24.3-47.3 \\
53.9\end{array}$ & 0.17 & 0.40 & 1.9 & 6.4 & C, Ch-2, V \\
\hline 9 & W10q13 & $100-19$ & $\begin{array}{l}44.5-61.4 \\
53.9\end{array}$ & -0.44 & $\begin{array}{l}-0.42 \\
-0.85\end{array}$ & 11.8 & 5.1 & $\mathrm{~B} 3, \mathrm{Cn}, \mathrm{K \tau}, \mathrm{MIr}, \mathrm{v}, \mathrm{W}$ \\
\hline 10 & $W 3 q 11$ & $1-65$ & $\begin{array}{l}47.0-58.9 \\
25.8 \\
12.0-39.6\end{array}$ & 0.18 & 0.72 & 2.4 & 5.0 & \\
\hline 10 & $E g q 7$ & $1-65$ & $\begin{array}{l}27.7 \\
17.8-39.6\end{array}$ & 0.21 & -0.52 & 4.6 & 6.4 & \\
\hline 10 & W6q6 & $1-65$ & $\begin{array}{l}29.7 \\
22.8-40.6\end{array}$ & 0.27 & -0.18 & 5.4 & 10.2 & \\
\hline 10 & W10q5 & $1-65$ & $\begin{array}{l}31.7 \\
24.8-42.6\end{array}$ & 0.21 & -0.76 & 5.3 & 10.2 & \\
\hline 10 & $W 10 q 8$ & $65-35$ & $\begin{array}{l}48.7 \\
45.5-55.3\end{array}$ & 0.17 & -0.59 & 2.8 & 8.9 & $\mathrm{Ch}, \mathrm{Cl}, \mathrm{V}$ \\
\hline 10 & $\operatorname{Lgaq5}$ & $65-35$ & $\begin{array}{l}51.4 \\
29.7-60.6\end{array}$ & 0.02 & -12.4 & 3.0 & 3.4 & $\mathrm{Ch}, \mathrm{Cl}, \mathrm{V}$ \\
\hline 10 & $W 6 q 8$ & $65-35$ & $\begin{array}{l}52.6 \\
45.5-57.9\end{array}$ & 0.24 & 0.08 & 3.0 & 9.2 & $\mathrm{Ch}, \mathrm{Cl}, \mathrm{V}$ \\
\hline 10 & $E g q 9$ & $65-35$ & $\begin{array}{l}52.6 \\
45.4-59.8\end{array}$ & 0.18 & 0.03 & 2.3 & 5.1 & $\mathrm{Ch}, \mathrm{Cl}, \mathrm{V}$ \\
\hline 11 & $W 3 q 4$ & $2-4$ & $\begin{array}{l}12.6 \\
2.4-22.8\end{array}$ & 0.21 & 0.53 & 3.0 & 9.5 & $\mathrm{~B}-3, \mathrm{Ch}, \mathrm{H}, \mathrm{K}, \mathrm{Mr}$ \\
\hline
\end{tabular}

(Continued) 
Table 3. Continued

\begin{tabular}{|c|c|c|c|c|c|c|c|c|}
\hline$M M U$ & Symbol & Flanking Markers ${ }^{\mathrm{b}}$ & Position $^{\mathrm{c}}(\mathrm{cM})$ & $a^{\mathrm{d}}$ & $d^{\mathrm{e}}$ & $\% \mathrm{~V}^{\mathrm{f}}$ & $L O D$ & Other studies ${ }^{\mathrm{g}}$ \\
\hline 11 & Lgaq1 & $2-4$ & $\begin{array}{r}12.6 \\
?-26.7\end{array}$ & 0.21 & 0.13 & 2.6 & 5.3 & $\mathrm{Ch}, \mathrm{H}, \mathrm{K}, \mathrm{Md}, \mathrm{V}$ \\
\hline 11 & $W 10 q 2$ & $2-4$ & $\begin{array}{l}17.7 \\
11.3-24.1\end{array}$ & 0.33 & -0.00 & 8.1 & 26.4 & B-3, Ch, H, K, Md, Mr, V \\
\hline 11 & $\operatorname{Lgq1}$ & $2-4$ & $\begin{array}{r}17.7 \\
?-30.5\end{array}$ & 0.19 & -0.08 & 2.7 & 5.5 & Ch, H, K, Md, P, V \\
\hline 11 & W6q3 & $2-4$ & $\begin{array}{l}20.3 \\
12.6-30.5\end{array}$ & 0.28 & 0.04 & 4.4 & 14.3 & $\mathrm{~B}-3, \mathrm{Ch}, \mathrm{H}, \mathrm{K}, \mathrm{Kt}, \mathrm{Md}, \mathrm{Mr}, \mathrm{P}, \mathrm{V}$ \\
\hline 11 & Tlq1 & $2-4$ & $\begin{array}{l}22.8 \\
13.9-31.8\end{array}$ & 0.22 & 0.72 & 3.8 & 10.3 & \\
\hline 11 & $\operatorname{Egq4}$ & $2-4$ & $\begin{array}{l}28.0 \\
16.5-43.0\end{array}$ & 0.21 & -0.25 & 2.7 & 8.5 & B-2-3, C, Ch, H, K, Kt, Lt, Md, P, V \\
\hline 12 & Tlaq1 & $5-8$ & $\begin{array}{l}48.8 \\
44.4-56.2\end{array}$ & -0.32 & -0.03 & 3.7 & 9.9 & Ch2 \\
\hline 12 & $W 6 q 10$ & $5-8$ & $\begin{array}{l}53.3 \\
45.9-?\end{array}$ & 0.17 & 0.70 & 1.9 & 6.4 & B2-3, Ch, Md, R, V \\
\hline 12 & Egq10 & $5-8$ & $\begin{array}{l}54.8 \\
45.9-?\end{array}$ & 0.15 & 0.53 & 1.5 & 5.0 & B2-3, Ch, Md, R, V \\
\hline 13 & Egaq4 & $27-53$ & $\begin{array}{l}35.0 \\
28.1-46.8\end{array}$ & 0.07 & -2.4 & 2.3 & 3.7 & B2-3, Ch, I, Kt, Ms \\
\hline 13 & $W 6 q 16$ & $21-53$ & $\begin{array}{l}49.5 \\
37.1-60.6\end{array}$ & 0.15 & -0.73 & 1.9 & 3.6 & B2-3, I, Kt, V \\
\hline 13 & Tlq3 & $21-53$ & $\begin{array}{l}54.1 \\
46.2-?\end{array}$ & 0.22 & 0.21 & 3.4 & 7.1 & \\
\hline 13 & Tlaq3 & $21-53$ & $\begin{array}{l}54.1 \\
46.2-?\end{array}$ & 0.21 & 0.33 & 2.2 & 4.1 & \\
\hline 13 & $W 10 q 17$ & $21-53$ & $\begin{array}{l}55.4 \\
45.6-?\end{array}$ & 0.13 & 0.38 & 1.0 & 4.2 & B3, I, Kt, V \\
\hline 15 & Tlaq4 & $13-1$ & $\begin{array}{r}21.0 \\
?-35.3\end{array}$ & 0.20 & 0.26 & 2.6 & 3.7 & $\mathrm{Ag}$ \\
\hline 15 & Tlq6 & $13-1$ & $\begin{array}{l}27.1 \\
10.8-40.4\end{array}$ & 0.16 & 0.54 & 2.1 & 3.5 & $\mathrm{Ag}$ \\
\hline 15 & $W 3 q 7$ & $13-1$ & $\begin{array}{l}35.3 \\
25.1-46.3\end{array}$ & 0.18 & 0.37 & 1.9 & 5.6 & $\mathrm{~A} 2, \mathrm{Ch}, \mathrm{Mr}, \mathrm{P}, \mathrm{R}, \mathrm{V}, \mathrm{Y}$ \\
\hline 15 & W3q6 & $1-15$ & $\begin{array}{l}49.4 \\
46.3-53.1\end{array}$ & 0.17 & 0.40 & 2.1 & 5.6 & $\mathrm{~A} 2, \mathrm{Ch}, \mathrm{Mr}, \mathrm{P}, \mathrm{R}, \mathrm{V}$ \\
\hline 17 & W6q11 & Ped-124 & $\begin{array}{r}19.2 \\
?-23.9\end{array}$ & 0.17 & -0.38 & 1.9 & 6.3 & Ch-2, Kt, Md, V \\
\hline 17 & W10q12 & Ped-124 & $\begin{array}{r}19.2 \\
?-22.6\end{array}$ & 0.15 & 0.01 & 2.1 & 6.9 & Ch-2, Kt, Md, V \\
\hline 17 & $\operatorname{Egq5}$ & Ped-124 & $\begin{array}{r}19.2 \\
?-23.9\end{array}$ & 0.18 & -0.47 & 2.4 & 7.7 & Ch2, Kt, V \\
\hline 17 & Egaq2 & Ped-124 & $\begin{array}{r}21.8 \\
?-23.9\end{array}$ & 0.16 & -0.25 & 1.8 & 5.5 & $\mathrm{Ch} 2, \mathrm{Kt}, \mathrm{V}$ \\
\hline 17 & $W 10 q 15$ & $10-76$ & $\begin{array}{l}30.9 \\
24.5-41.1\end{array}$ & 0.14 & 0.19 & 3.1 & 4.8 & Ch2, V \\
\hline 17 & Egaq3 & $10-76$ & $\begin{array}{l}33.7 \\
23.9-44.6\end{array}$ & 0.17 & -0.28 & 2.5 & 3.9 & $\mathrm{Ch} 2, \mathrm{~V}$ \\
\hline 17 & W6q12 & $10-76$ & $\begin{array}{l}35.1 \\
23.9-43.1\end{array}$ & 0.20 & -0.46 & 3.8 & 5.6 & Ch2, V \\
\hline 17 & Egq6 & $10-76$ & $\begin{array}{l}35.1 \\
23.9-41.6\end{array}$ & 0.21 & -0.67 & 4.7 & 6.6 & Ch2, V \\
\hline 18 & $W 3 q 15$ & $19-51$ & $\begin{array}{l}29.4 \\
15.7-37.0\end{array}$ & 0.12 & 1.1 & 1.6 & 3.7 & A2, Ch-2, T, V \\
\hline 18 & $W 3 q 16$ & $51-3$ & $\begin{array}{l}39.4 \\
37.0-46.0\end{array}$ & 0.10 & 1.2 & 1.3 & 3.6 & $\mathrm{~A} 2, \mathrm{Ch}, \mathrm{P}, \mathrm{V}$ \\
\hline
\end{tabular}


Table 3. Continued

\begin{tabular}{lcccccccc}
\hline MMU & Symbol & Flanking Markers $^{\mathrm{b}}$ & Position $^{\mathrm{c}}(c M)$ & $d^{\mathrm{d}}$ & $a^{\mathrm{e}}$ & $\% \mathrm{~V}^{\mathrm{f}}$ & LOD & Other studies $^{\mathrm{g}}$ \\
\hline 19 & W3q14 & $45-36$ & 36.3 & 0.15 & 0.06 & 0.8 & 3.7 & $\mathrm{Kl}, \mathrm{P}$ \\
& & $17.0-?$ & & & & & \\
\hline
\end{tabular}

${ }^{a}$ W3qi, W6qi, W10qi, Tlqi, Egqi, Lgqi, Tlaqi, Egaqi, and Lgaqi are symbols attributed to QTL detected for 3-week weight (WT3wk), 6week weight (WT6wk), 10-week weight (WT10wk), tail length (TAIL), growth between weeks 3 and 6 (EARLYGR), growth between weeks 6 and 10 (LATEGR), TAIL adjusted for WT6wk, EARLYGR adjusted for WT3wk, and LATEGR adjusted for WT6wk. Numeric indices (i) within trait reflect descending rank of maximum LOD scores.

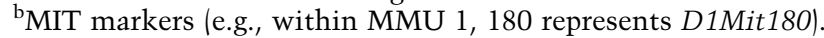

${ }^{\mathrm{c} A p p r o x i m a t e}$ positions (Mouse Genome Database) of maximum likelihood peaks (top) and respective one LOD confidence intervals (bottom). A '?' indicates that a confidence interval extends to the beginning or end of a chromosome.

${ }^{\mathrm{d}}$ Additive effect (Falconer and Mackay 1996) in phenotypic S.D. units. Negative values indicate increasing effect of the $L 6$ allele.

'Degree of relative dominance: 0 indicates additivity, 1 full dominance of the M16i allele, and -1 full dominance of the $L 6$ allele.

fercentage of phenotypic variance accounted for by QTL.

${ }^{g}$ Studies detecting QTL for similar traits in the same genomic region, listed in order of location on each chromosome (from proximal to distal). A and A2 are Anunciado et al. (2000, 2001); Ag, Agulnik et al. (1998); B, B2, and B3 are Brockmann et al. (1998a, 1998b, 2000); C, Corva et al. (2001); Ch and Ch2, Cheverud et al. (1996, 2001); Cl, Collins et al. (1993); D, Drudik et al. (1995); H, Hirayama et al. (1999); I, Ishikawa et al. (2000); K, Kirkpatrick et al. (1998); Kl, Kluge et al. (2000); Kt, Keightley et al. (1996); L, Lembertas et al. (1997); Lt, Leiter et al. (1998); M, Mehrabian et al. (1998); Md, Moody et al. (1999); Mr, Morris et al. (1999); Ms, Masinde et al. (2002); P, Pomp et al. (1994); Pl, Plum et al. (2000); R, Reifsnyder et al. (2000); T, Taylor et al. (1999); V, Vaughn et al. (1999); W, West et al. (1994); and Y, York et al. (1996).

Table 4. Summary of QTL results across traits: gene action

\begin{tabular}{lccccrrr}
\hline Trait $^{\mathrm{a}}$ & \#QTL $^{\mathrm{b}}$ & \#Chr & Avg. $a^{\mathrm{c}}$ & Max. $a$ & Min. $a$ & Avg. $d^{\mathrm{d}}$ & Max. $d$ \\
\hline WT3wk & 17 & 12 & 0.18 & 0.42 & 0.10 & 0.73 & 1.2 \\
WT6wk & 17 & 12 & 0.23 & 0.56 & 0.12 & 0.08 & 0.70 \\
WT10wk & 18 & 13 & 0.21 & 0.35 & 0.12 & 0.03 & 0.66 \\
TAIL & 6 & 5 & 0.19 & 0.22 & 0.13 & 0.41 & -0.73 \\
TAILadj & 4 & 4 & 0.29 & 0.21 & -0.44 & 0.37 & 0.72 \\
EARLYGR & 12 & 8 & 0.21 & 0.42 & 0.14 & -0.15 & 0.08 \\
EARLYadj & 4 & 3 & 0.14 & 0.17 & 0.07 & -0.88 & -0.25 \\
LATEGR & 6 & 5 & 0.17 & 0.15 & 0.19 & -0.25 & -0.08 \\
LATEadj & 5 & 5 & 0.16 & 0.21 & 0.02 & -2.7 & -0.67 \\
\hline
\end{tabular}

3-week weight, 6-week weight, 10-week weight, tail length, growth from weeks 3 to 6 , growth from weeks 6 to 10, TAIL adjusted for WT6wk, EARLYGR adjusted for WT3wk, and LATEGR adjusted for WT6wk.

${ }^{\mathrm{b}}$ Number of QTL, and number of chromosomes in which QTL were detected for the trait.

${ }^{\mathrm{c}}$ Average of absolute values of $a$.

${ }^{\mathrm{d}}$ Average of degree of directional dominance (not average of absolute values of $d$ ).

QTL genotypes considerably slows with increasing maturity relative to the rate of growth of individuals with multiple homozygous QTL genotypes. These estimates are magnified when adjusted for initial body weight (Table 4). The distribution of estimates of QTL additive effects exhibits an exponential profile (Fig. 2).

For all QTL detected, M16i alleles had an additive effect, increasing weight and growth as expected (Table 3), with the exception of TAILADJ. Effects of considerable magnitude were detected on Chr 2 (Fig. 3) for all body weight traits, with evidence for multiple QTL in the distal region.

Congruencies of QTL for different growth traits are displayed in Table 2 and roughly approximate the extent of overlap between QTL detected for different traits. Congruencies of QTL for WT3wk and WT6wk, WT3wk and WT10wk, and EARLYGR and
LATEGR are considerably lower than that associating WT6wk and WT10wk. This indicates the extent to which different QTL are responsible for growth during different age periods (illustrated in Fig. 4). In general, QTL Congruencies mirrored phenotypic correlations.

No conclusive statistical evidence of QTL by sex interaction was found. No sex X QTL-linked marker interactions were significant in the marker-based models. When CIM was implemented by gender subsets, sets of single-sex significant QTL were for the most part largely coincident between males and females, with reasonably similar estimates of additive and dominance effects across sexes.

With no epistasis, the total fraction of phenotypic variance accounted for by all detected QTL when fitted together should approximate the sum of the individual QTL variance effects. For some traits 

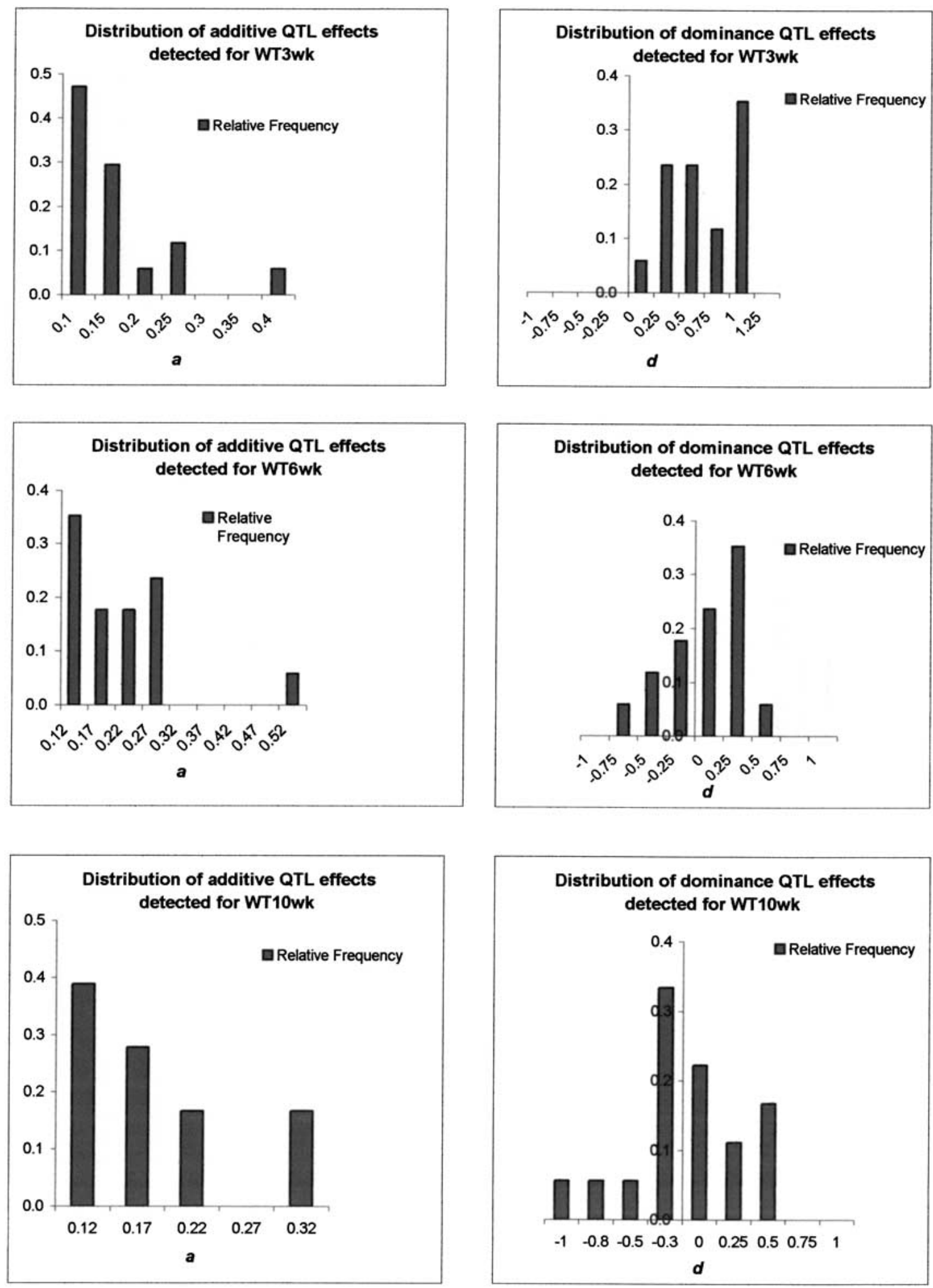

Fig. 2. Relative frequencies of magnitudes of estimates of additive and dominance QTL effects for WT3wk, WT6wk, and WT10wk. Magnitudes of additive effects are standardized in phenotypic S.D. units. Magnitudes of dominance effects are degrees of relative dominance, with 0 indicating additivity; 1 , full dominance of the M16i allele; and -1 indicating full dominance of the $L 6$ allele. 


\section{Chromosome 2 - Composite Interval Mapping}

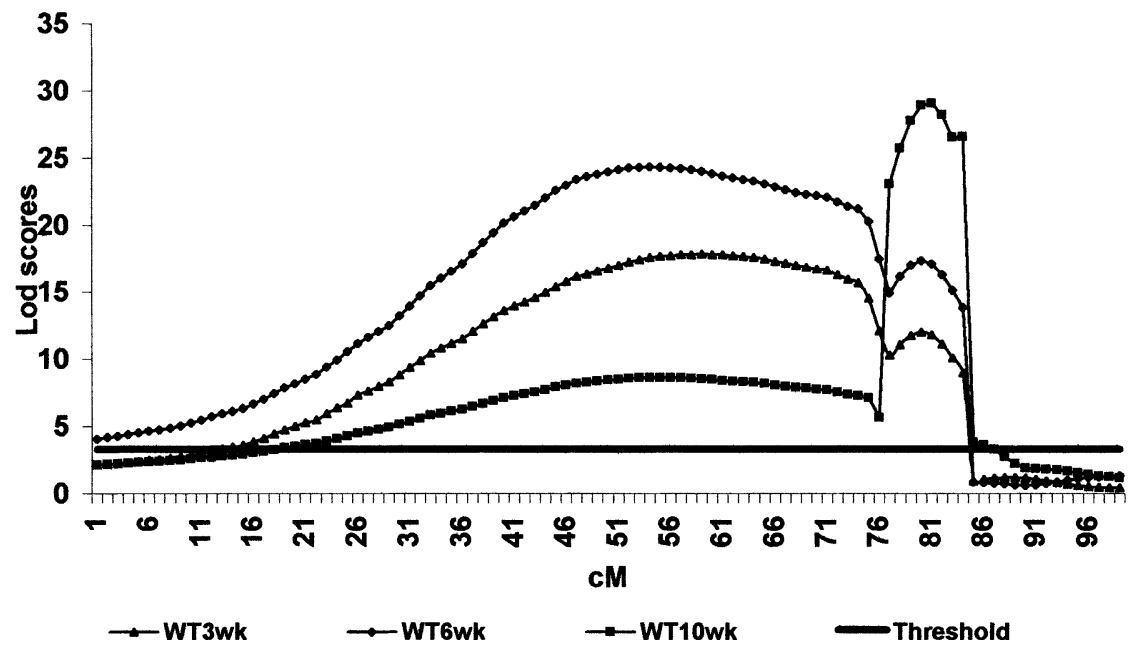

Fig. 3. Chromosome 2 QTL likelihood plots from Composite Interval Mapping for WT3wk, WT6wk, and WT10wk. this expectation appeared to be verified (Table 5), but that was not the case for WT6wk and especially WT3wk, for which no difference existed between the total fraction of the phenotypic variance accounted for by the 5 QTL with largest and 5 QTL with smallest effects.

Pair-wise interactions were detected for some growth traits. QTL for WT6wk on MMU6 interact with those on MMU17 $(P=.0014$; Figure 5a,b), while QTL for WT10wk on MMU3 interact with those on MMU5 $(P=.011$; Figure $5 \mathrm{c}, \mathrm{d})$. The interpretation of these interactions is limited by the fact

Timeline of Chromosomal Participation in the Biological Processes of Growth

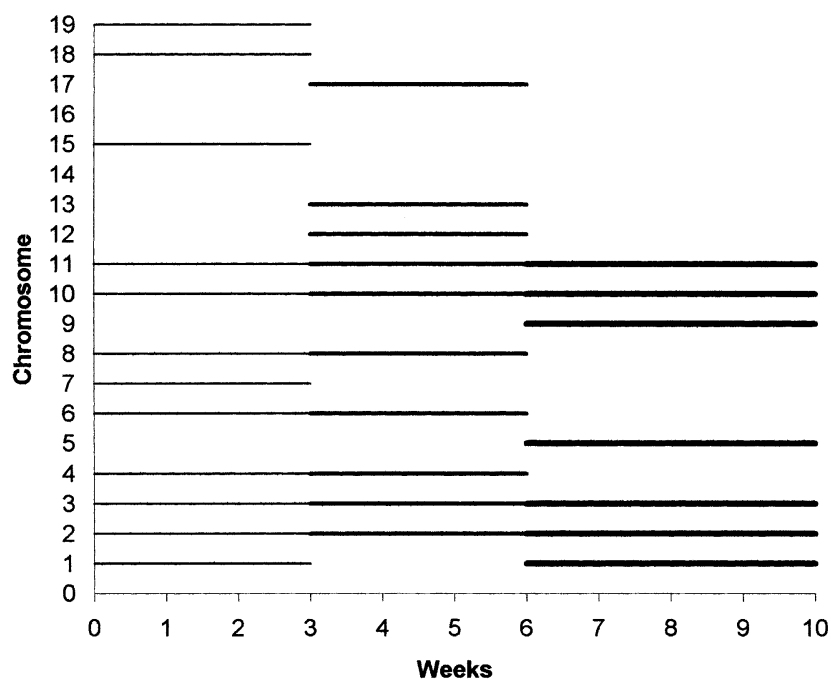

Fig. 4. Involvement of mouse chromosomes in growth predisposition at the three periods measured $(0-3$ weeks, 3-6 weeks, and $6-10$ weeks). that they are evaluated from linked-marker effects, and there are two linked QTL impacting the marker-effect involved in the interactions in the cases of MMU6, MMU17, and MMU3 (Table 3). For the WT6wk interaction, the presence of either L6 allele appears to attenuate the impact of the M16i allele of the QTL from the other chromosome. This is particularly so for the MMU6 L6 alleles which, when present in a homozygous form, result in no differentiation among the MMU17 genotypes (common origin for all three lines in Fig. 5b). The WT10wk interaction consists of a change in the dominance relationships of the QTL of each chromosome across the genotypes of the QTL of the other chromosome. Homozygosity of L6 at MMU3 renders the L6 allele of MMU5 fully dominant, while with at least one MMU3 M16i allele present, the MMU5 M16i allele is partially dominant (Fig. 5d).

Genomic and chromosomal polygenic analyses. For all three body weight traits, practically no gains in model $\mathrm{R}^{2}$ are obtained from adding additional markers once selected markers are accounted for (data not shown). These comparisons are among models with the same degrees of freedom and seem to indicate that large regions of the genome may contribute little to the phenotypic variation of these traits, discounting the existence of a large number of very small effects with minimal impacts. An interesting byproduct of these analyses relates to previous results on dominance effects. As age of the mice increases, the regression coefficient associated with the percentage of the genome in a heterozygous state (i.e., grams per percent of genome in heterozygous state) drops significantly from WT3wk to WT6wk 


\section{a - Single Marker Effects (WT6wk)}

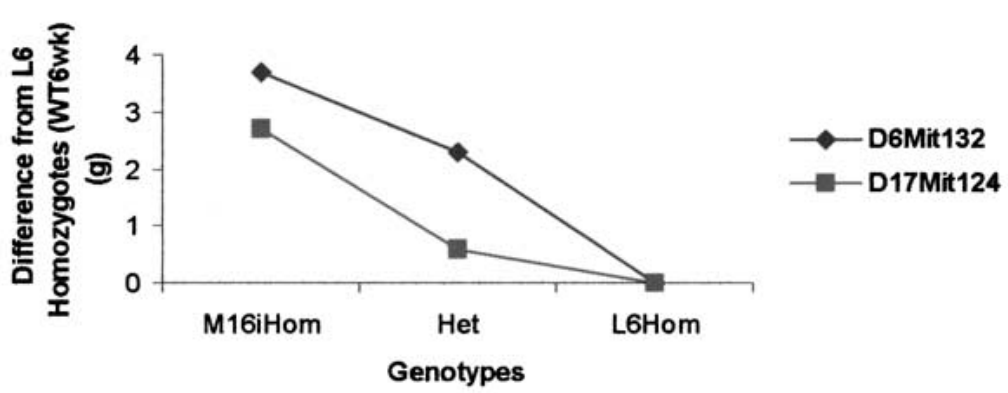

b - Interaction between QTL on MMU6 and MMU17 (WT6wk)

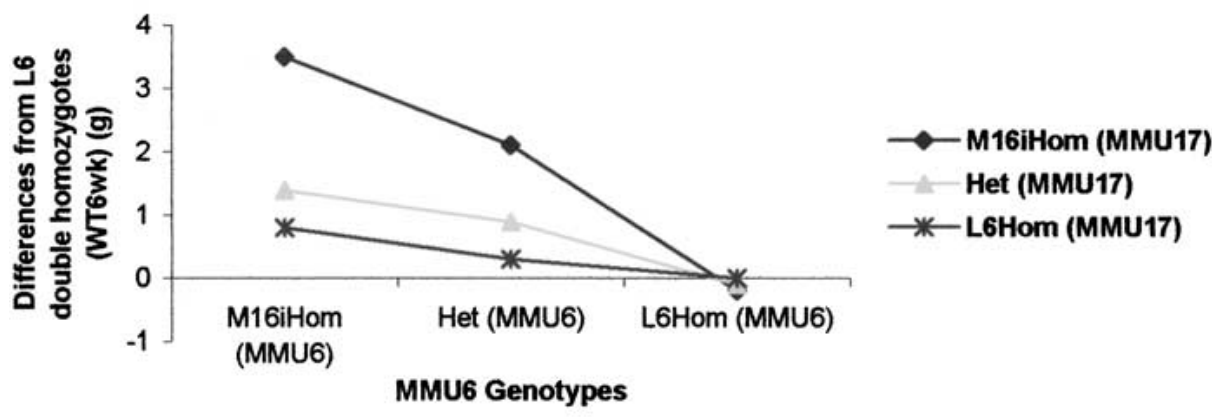

c - Single Marker Effects (WT 10wk)

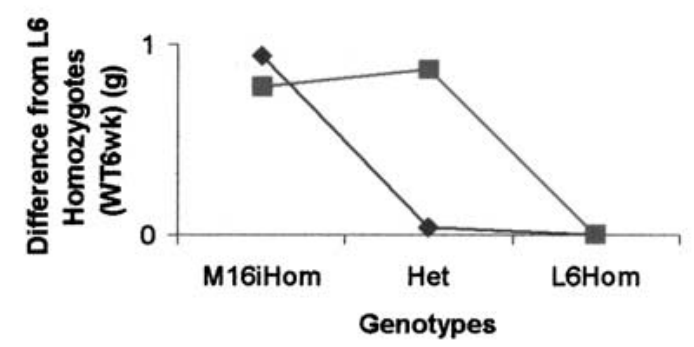

\section{d - Interaction between QTL on MMU3 and MMU5 (WT10wk)}

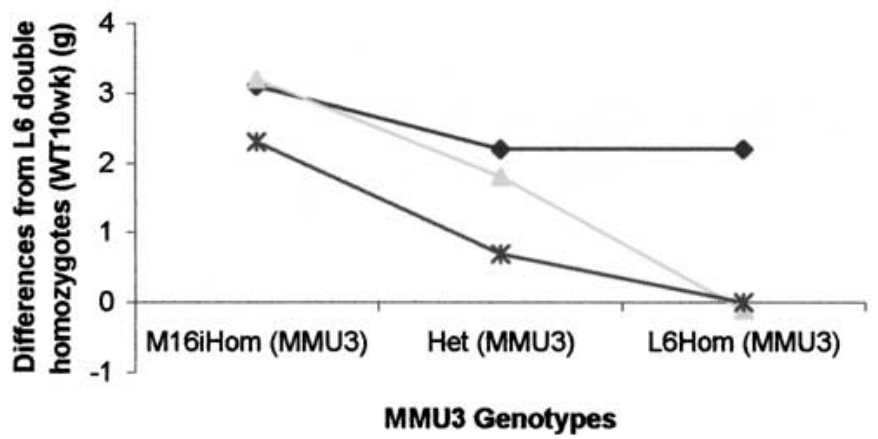

Fig. 5. Epistatic interactions between QTL evaluated from linkedmarker effects. a and c: single marker effects; $\mathbf{b}$ and $\mathbf{d}$ : marker interactions for, respectively, WT6wk and WT10wk. 
Table 5. Summary of QTL results across traits: gene effects

\begin{tabular}{|c|c|c|c|c|c|c|c|}
\hline Trait $^{\mathrm{a}}$ & Avg \% Var & Max \% Var & Min \% Var & Total \% Var & First $5 \%$ Var $^{\mathrm{c}}$ & Last $5 \% \operatorname{Var}^{\mathrm{d}}$ & $A v g . C I^{\mathrm{e}}$ \\
\hline WT3wk & 2.9 & 13.2 & 0.8 & $-\overline{(79.7)}$ & 19.9 & 21.1 & $\begin{array}{l}17.9 \\
(28.2-6.8)\end{array}$ \\
\hline WT6wk & 5.1 & 24.2 & 1.5 & (104.1) & 36.2 & 10.5 & $\begin{array}{l}18.7 \\
(31.5-5.6)\end{array}$ \\
\hline WT10wk & 5.8 & 23.6 & 1.0 & $-\overline{(127.7)}$ & 33.6 & 10.0 & $\begin{array}{l}17.1 \\
(32.4-4.9)\end{array}$ \\
\hline TAIL & 2.5 & 3.8 & 1.7 & $\begin{array}{l}13.1 \\
(26.6)\end{array}$ & - & & $\begin{array}{l}16.3 \\
(29.6-6.0)\end{array}$ \\
\hline TAILadj & 5.1 & 11.8 & 2.2 & $\begin{array}{l}19.4 \\
(-)\end{array}$ & - & - & $\begin{array}{l}11.9 \\
(11.8-11.9)\end{array}$ \\
\hline EARLYGR & 4.2 & 13.9 & 1.1 & $\overline{(65.2)}$ & 23.3 & 10.0 & $\begin{array}{l}18.8 \\
(26.5-7.0)\end{array}$ \\
\hline EARLYadj & 2.4 & 2.9 & 1.8 & $\begin{array}{l}5.8 \\
(-)\end{array}$ & - & - & $\begin{array}{l}22.3 \\
(27.4-18.7)\end{array}$ \\
\hline LATEGR & 2.6 & 2.9 & 2.2 & $\begin{array}{l}12.3 \\
(28.2)\end{array}$ & - & - & $\begin{array}{l}26.1 \\
(37.1-15.0)\end{array}$ \\
\hline LATEGRadj & 2.7 & 3.2 & 2.2 & $\begin{array}{l}13.4 \\
(-)\end{array}$ & - & - & $\begin{array}{l}23.9 \\
(30.9-16.9)\end{array}$ \\
\hline
\end{tabular}

3-week weight, 6-week weight, 10-week weight, tail length, 3- to 6-week growth, 6- to 10- weeks growth, TAIL adjusted for WT6wk, EARLYGR adjusted for WT3wk, and LATEGR adjusted for5 WT6wk.

${ }^{\mathrm{b}}$ Total percentage of phenotypic variance accounted for by all QTL detected for a trait. For some traits the large number of QTL detected precluded estimation of this statistic. In parentheses is the percentage of the F2 range accounted for when additive effects (2a) of all QTL detected for a trait are summed.

${ }^{\mathrm{c}}$ Total percentage of phenotypic variance accounted for by largest 5 QTL.

${ }^{\mathrm{d}}$ Total percentage of phenotypic variance accounted for by smallest 5 (or remaining) QTL.

${ }^{\mathrm{e}}$ Average length of the one LOD confidence intervals defined for the trait QTLs (situationswhere one of the CI boundaries could not be defined are excluded from this average). In parentheses are the corresponding maximum and minimum lengths of such CIs.

and becomes nonsignificant for WT10wk (data not shown), indicating a decrease in the importance of dominance with increasing maturity.

\section{Discussion}

Early growth traits, representing an important component of fitness (Cheverud et al. 1996), often exhibit appreciable levels of heterosis with strong directional dominance (Falconer and Mackay 1996). As ontogeny progresses and growth phenotypes emerge from maternal influences, their heritabilities increase (Malik 1984), and often heterosis becomes less important (White et al. 1970; Eisen et al. 1977; HorstgenSchwark et al. 1984), indicating a shift in the profiles of directional dominance associated with the genetic architecture of the maturing organism. Our results fully support this scenario. Additional insights that this study provides, and that classical theory cannot offer, are that the shift in the profiles of directional dominance towards aggregate additivity with increasing maturity does not come from uniform trends towards individual additivity across all loci impacting the growth traits, but rather from a reversal of the direction of dominance for many loci. Similar results can be found in the studies of Cheverud et al. (1996) and Vaughn et al. (1999), who used the LG and SM inbred lines derived from strains selected for large and small size. The same patterns can be seen for the dominance results reported by two other independent QTL studies for growth (Morris et al. 1999; Moody et al. 1999). Validated and verified by five different studies, and previously hinted at by Dickerson (1973) for beef cattle and by other studies in mice (White et al. 1970; Eisen et al. 1977; Horstgen-Schwark et al 1984), this finding appears to have biological validity and significance, and the underlying physiological basis merits further study.

While some previous large QTL studies have focused on a single end-point measurement of growth (e.g., Brockmann et al. 1998b, 2000), others (Cheverud et al. 1996; Vaughn et al. 1999) have conducted comprehensive experiments evaluating growth at different age periods. Although the sample size used by Cheverud et al. (1996) was smaller ( $n=$ 535), the Vaughn et al. (1999) study used an $\mathrm{F}_{2}$ population with power similar to that in the present study $(n=1045)$. Cheverud et al. (1996) identified 16 QTL for WT3wk, 15 for WT6wk, and 17 for WT10wk, numbers very similar to those we identified. In addition, QTL map locations from the two studies coincide extremely well, and estimates of average additive, dominance, and variance QTL effects were similar. The study of Vaughn et al. (1999) 
encompassed and expanded the experimental sample used by Cheverud et al. (1996), and both sets of results were similar in all respects. Another reference study of QTL for mouse growth was that of Keightley et al. (1996), and the coincidence of QTL map locations from their study with our results was excellent in many instances. Coincidence of QTL map locations was also observed for some of the lean body mass QTL reported by Masinde et al. (2002). Corva and Medrano (2001) and Brockmann and Bevova (2002) have made comprehensive and detailed reviews of many growth QTL studies in the mouse, and our results integrate well within this framework (see Table 3 for an updated review of mouse growth QTL in reference to our findings).

Some of the effects detected on distal Chr 2 have a remarkable magnitude, and the potential biological significance of a thorough genomic dissection of these loci is evident. Efforts to fine-map this gene (or genes) are being actively pursued in our Lab using congenic lines and integrated transcriptional, proteomic, and metabolomic approaches (Pomp et al. 2002). Findings of QTL for growth and obesity in this region match results from many other studies (see listing in Table 3), although estimates of effects appear to be maximized in crosses with the M16i selection line. In addition to the Chr 2 effects, Chrs 1 , $3,6,10,11$, and 17 emerged as the most relevant in contributing to phenotypic variation for growth in this study.

The general lack of many significant epistatic interactions in this cross is in contrast to results of several studies (e.g., Routman and Cheverud 1997; Brockmann et al. 2000). However, this finding is not unusual (Clark 2000), as attempts to detect epistasis with these types of linear models raise a number of issues of difficult resolution (e.g., Wade 1992; Cheverud 2000; Goodnight 2000; Nelson et aL 2001). The lack of statistical detection of epistatic interactions in the context of these models does not preclude the existence or the importance of epistasis, as our results (especially for WT3wk) seem to confirm. A more thorough dissection of these issues would require the implementation of multiple QTL-models (Kao et al. 1999) or of the alternative approaches proposed by Routman and Cheverud (1997). We plan to undertake these in the future.

For the WT6wk epistatic interaction, a buffering effect appears to be occurring between the loci from the two chromosomes such that the presence of either L6 allele attenuates the impact of the M16i allele at the QTL from the other chromosome. This is particularly so for the MMU6 L6 alleles which, when present in a homozygous form, mask differentiation among MMU17 genotypes. Interestingly, an epi- static interaction of a similar nature between QTL on the same two chromosomes was reported for body wt at 12 weeks by Reifsnyder et al. (2000). Replication of specific findings of epistasis across studies provides greater evidence that the interaction is real and not a statistical artifact.

Substantial evidence for gender-specific QTL was lacking in this study, in contrast to other studies (e.g., Vaughn et al. 1999). However, this result is also not unusual (Brockmann et al. 1998b), and statistical issues involved in detection of such interactions require careful interpretation. Our conclusion that early and late growth are influenced by fairly different sets of genes (i.e., low QTL congruency) was according to expectations and previous observations /Cheverud et al. 1996; Vaughn et al. 1999), and parallel trends in the phenotypic correlations among these traits.

Multiple-trait QTL analyses (Jiang and Zeng 1995) were also conducted, and results will be published separately. In short, they enabled some additional resolution and power, but the value of these improvements was minimal relative to the effort involved. Certainly many of the individual QTL that were identified in the same region for correlated traits represent pleiotropic effects of single QTL, but discerning pleiotropy from closely linked independent QTL is a difficult task. Furthermore, estimates of QTL effects and variance contributed may be biased by the presence of two or more linked QTL in cis (coupling), which is likely as a consequence of the long-term selection history of the parental lines used in this study.

The impact of basing QTL analyses on residuals from preliminary models, which pre-adjust data for fixed effects and covariates, was evaluated. Interestingly, we found that this strategy, which has been adopted for use in many previous studies, introduces a consistent downward bias (between 10\% and 20\%) of the magnitude of most estimates of QTL effects (data not shown). Given the upward biases that have been recognized to be associated with the implementation of QTL analyses (e.g., Beavis 1998; Goring et al. 2001; Allison et al. 2002), the present results are not of particular concern. Besides a slight reduction in the statistical power of the experiment, no negative impacts of this strategy could be identified.

When results of our study, with large sample size and multi-trait characteristics, are integrated with those of previous studies to provide a general understanding of QTL detection and the genetic architecture of growth, several primary conclusions emerge. First, numbers of QTL and magnitudes of individual effects detected are clearly sample-size dependent. Small experiments can lead to large 
estimates of QTL effects that are usually the result of statistical biases (Beavis 1998; Melchinger et al 1998; Young 1999), while experiments of larger sample size considerably shrink the magnitude of estimates of effects detected in smaller experiments. Second, the distribution of QTL effects clearly does not conform with the uniform distribution proposed in the context of the infinitesimal model, but rather approximates an exponential model [as predicted by Robertson (1967) and shown by others (Bost et al. 1999; Hayes and Goddard 2001; Mackay 2001)] that nonetheless maintains an infinitesimal quality (Jinks 1977). Third, the primary contributions to genetic control of growth derive from localized genomic regions, and not from the genome as a whole, with vast genomic regions excluded. Knott et al. (1998) obtained similar results. Fourth, agreement observed between most of the results concerning dominance and theoretical expectations determined by principles of classical quantitative genetics, as well as consistency observed for locations of many detected QTL across a wide variety of studies with different genetic backgrounds, is impressive and bears witness to the soundness of the statistical methodology that has been developed to support and implement QTL analyses.

\section{Acknowledgements}

We gratefully acknowledge Mary Ann Cushman, Stephenie Foster, and Grady Beck for collection of genotypic data. We appreciate the assistance of James Specht and Steven Kachman in statistical analyses and use of QTL detection computer packages. We have also benefited greatly from useful discussions with Jerry Taylor, Mark Thallman, and Gary Rohrer, and from constructive reviews of a previous version of this manuscript by Bill Hill and Merlyn Nielsen. J.L. Rocha acknowledges the support of the Portuguese Foundation for Science and Technology. This research is a contribution of the University of Nebraska Agricultural Research Division (Lincoln, nab.; Journal Series No. 14110) and the North Carolina Agricultural Research Service, and was supported in part by funds provided through the Hatch Act. This research was also partially based upon work supported by the National Science Foundation under Grant No. 0091900 (Nebraska EPSCOR infrastructure improvement grant).

\section{References}

1. Agulnik II, Agulnik SI, Saatkamp BD, Silver LM (1998) Sex-specific modifiers of tail development in mice heterozygous for the brachyury (T) mutation. Mamm Genome 9, 107-110

2. Allison DB, Fernandez JR, Heo M, Zhu S, Etzel C et al. (2002) Bias in estimates of quantitative-trait-locus effect in genome scans: demonstration of the phenomenon and a method-of-moments procedure for reducing bias. Am J Hum Genet 70, 575-585

3. Andersson L (1998) Identification and cloning of trait genes. In Animal Breeding Technology for the $21^{\text {st }}$ Century, AJ Clark, ed. (Amsterdam, The Netherlands: Harwood Academic Publishers), pp 103-117

4. Anunciado RV, Ohno T, Mori M, Ishikawa A, Tanaka S et al. (2000) Distribution of body weight, blood insulin and lipid levels in the SMXA recombinant inbred strains and the QTL analysis. Exp Anim (Tokyo) 49, 217-224

5. Anunciado RV, Nishimura M, Mori M, Ishikawa A, Tanaka $S$ et al. (2001) Quantitative trait loci for body weight in the intercross between $\mathrm{SM} / \mathrm{J}$ and $\mathrm{A} / 3$ mice. Exp Anim (Tokyo) 50, 319-324

6. Atchley WR, Zhu J (1997) Developmental quantitative genetics, conditional epigenetic variability and growth in mice. Genetics 147, 765-776

7. Basten CJ, Weir BS, Zeng Z-B (2001) QTL Cartographer, version 1.15 (Raleigh, NC: North Carolina State University)

8. Beavis WD (1998) Power, precision, and accuracy. In Molecular Dissection of Complex Traits, AH Paterson, ed. (Boca Raton, Fla: CRC Press), pp 145-162

9. Bost B, Dillmann C, de Vienne D (1999) Fluxes and metabolic pools as model traits for quantitative genetics. I. The L-shaped distribution of gene effects. Genetics 153, 2001-2012

10. Botstein D, White RL, Skolnick M, Davis RW (1980) Construction of a genetic linkage map in man using restriction fragment length polymorphisms. Am J Hum Genet 32, 314-324

11. Brockinann GA, Bevova MR (2002) Using mouse models to dissect the genetics of obesity. Trends Genet $18,367-376$

12. Brockmann GA, Renne U, Kopplow K, Das P (1998a) Genetic markers for the detection of quantitative trait loci with special consideration of body weight and fat. Acta Theriol (Suppl 5), 53-62

13. Brockmann GA, Haley CS, Renne U, Knott SA, Schwerin M (1998b) Quantitative trait loci affecting body weight and fatness from a mouse line selected for extreme high growth. Genetics 150, 369-381

14. Brockmann GA, Kratzsch J, Haley CS, Renne U, Schwerin M et al. (2000) Single QTL effects, epistasis, and pleiotropy account for two-thirds of the phenotypic $\mathrm{F}_{2}$ variance of growth and obesity in DU6i x DBA/2 mice. Genome Res 10, 1941-1957

15. Carter TC, Falconer DS (1951) Stocks for detecting linkage in the mouse and the theory of their design. J Genet 50, 307-323

16. Cheverud JM (2000) Detecting epistasis among quantitative trait loci. In Epistasis and the Evolutionary Process, JB Wolf, ED Brodie III, MJ Wade, eds. (Oxford, UK: Oxford University Press), pp 58-81 
17. Cheverud JM, Routman EJ Duarte FA, van Swinderen B, Cothran K et al. (1996) Quantitative trait loci for murine growth. Genetics 142, 1305-1319

18. Cheverud JM, Vaughn TT, Pletscher LS, Peripato AC, Adams ES et al. (2001) Genetic architecture of adiposity in the cross of LG/J and SM/J inbred mice. Mamm Genome 12, 3-12

19. Churchill GA, Doerge RW (1994) Empirical threshold values for quantitative trait mapping. Genetics 138, 963-971

20. Clark AG (2000) Limits to prediction of phenotypes from knowledge of genotypes. Evol Biol 32, 205-224

21. Collins AC, Martin IC, Kirkpatrick BW (1993) Growth quantitative trait loci (QTL) on mouse chromosome 10 in a Quackenbush-Swiss $\times$ C57BL/6J backcross. Mamm Genome 4, 454-458

22. Corva PM, Medrano JF (2001) Quantitative trait loci (QTLs) mapping for growth traits in the mouse: a review. Genet Sel Evol 33, 105-132

23. Corva PM, Horvat S, Medrano JF (2001) Quantitative trait loci affecting growth in high growth (hg) mice. Mamm Genome 12, 284-290

24. Darvasi A, Soller M (1994) Optimum spacing of genetic markers for determining linkage between marker loci and quantitative trait loci. Theor Appl Genet 89, 351-357

25. Dickerson GE (1973) Inbreeding and heterosis in animals. In Proc Animal Breeding and Genetics Symposium in honor of Dr. Jay L. Lush (Champaign, Illinois: American Society of Animal Science), pp 54-77

26. Drudik DK, Pomp D, Zeng Z-B, Eisen EJ (1995) Identification of major genes controlling body weight and fat percentage on mouse chromosome 2. J Anim Sci 73 (Suppl 1), 110 (abst)

27. Eisen EJ, Bakker H, Nagai J (1977) Body composition and energetic efficiency in two lines of mice selected for rapid growth rate and their F1 crosses. Theor Appl Genet 49, 21-34

28. Falconer DS, Mackay TF (1996) Introduction to Quantitative Genetics (Harlow, UK: Longman Group Ltd.)

29. Frary A, Nesbitt TC, Frary A, Grandillo S, Knaap E et al. (2000) fw2.2: a quantitative trait locus key to the evolution of tomato fruit size. Science 289, 85-88

30. Georges M (1998) Mapping genes underlying production traits in livestock. In Animal Breeding Technology for the $21^{\text {st }}$ Century, AJ Clark, ed. (Amsterdam, The Netherlands: Harwood Academic Publishers), pp 77-101

31. Goodnight CJ (2000) Quantitative trait loci and gene interaction: the quantitative genetics of meta-populations. Heredity 84, 587-598

32. Goring HH, Terwilliger JD, Blangero J (2001) Large upward bias in estimation of locus-specific effects from genome-wide scans. Am J Hum Genet 69, 13571369

33. Green MC (1989) Catalog of mutant genes and polymorphic loci. In Genetic Variants and Strains of the Laboratory Mouse, MF Lyon, AG Searle, eds. (Oxford, UK: Oxford University Press), pp 12-403
34. Hackett CA (1997) Model diagnostics for fitting QTL models to trait and marker data by interval mapping. Heredity 79, 319-328

35. Hanrahan JP, Eisen EJ, Legates JE (1973) Effects of population size and selection intensity on short-term response to selection for post-weaning gain in mice. Genetics 73, 513-530

36. Hayes B, Goddard ME (2001) The distribution of the effects of genes affecting quantitative traits in livestock. Genet Sel Evol 33, 209-229

37. Hirayama I, Yi Z, Izumi S, Arai I, Suzuki W et al. (1999) Genetic analysis of obese diabetes in the TSOD mouse. Diabetes 48, 1183-1191

38. Horstgen-Schwark G, Eisen EJ, Saxton AM, Bandy TR (1984) Diallel cross among lines of mice selected for litter size and body weight:growth traits. Z Tierz Züchtungsbiol 101, 96-111

39. Ishikawa A, Matsuda Y, Namikawa T (2000) Detection of quantitative trait loci for body weight at 10 weeks from Philippine wild mice. Mamm Genome 11, 824-830

40. Jiang C, Zeng Z-B (1995) Multiple trait analysis of genetic mapping for quantitative trait loci. Genetics 140, 1111-1127

41. Jinks JL (1977) Discussion of Dr. Eaves' paper. J R Statist Soc Ser A 140, 352-353

42. Kao C-H, Zeng Z-B, Teasdale RD (1999) Multiple interval mapping for quantitative trait loci. Genetics 152, 1203-1216

43. Keightley PD, Knott SA (1999) Testing the correspondence between map positions of quantitative trait loci. Genet Res 74, 323-328

44. Keightley PD, Hardge T, May L, Bulfleld G (1999) A genetic map of quantitative trait loci for body weight in the mouse. Genetics 142, 227-235

45. Kirkpatrick BW, Mengelt A, Schulman N, Martin IC (1998) Identification of quantitative trait loci for prolificacy and growth in mice. Mamm Genome 9, 97-102

46. Kluge R, Giesen K, Bahrenberg G, Plum L, Ortlepp JR et al. (2000) Quantitative trait loci for obesity and insulin resistance (Nob1, Nob2) and their interaction with the leptin receptor allele $\left(\right.$ Lepr $\left.^{A 720 T / T 1044 I}\right)$ in New Zealand obese mice. Diabetologia 43, 15651572

47. Knott SA, Marklund L, Haley CS, Andersson K, Davies $\mathrm{W}$ et al. (1998) Multiple marker mapping of quantitative trait loci in a cross between outbred wild boar and Large White pigs. Genetics 149, 1069-1080

48. Lander ES, Botstein D (1989) Mapping Mendelian factors underlying quantitative traits using RFLP linkage maps. Genetics 121, 185-199

49. Legates JE (1969) Direct and correlated responses to selection in mice. In Genetics Lectures, vol. 1, R. Bogart, ed. (Corvallis, Oregon: Oregon State University Press), pp 149-165

50. Leiter EH, Reifsnyder PC, Flurkey K, Partke H-J, Junger E et al. (1998) Deleterious synergism by both parental genomes contributes to diabetogenic thresholds. Diabetes $47,1287-1295$ 
51. Lembertas AV, Perusse L, Chagnon YC, Fisler JS, Warden $\mathrm{CH}$ et al. (1997) Identification of an obesity quantitative trait locus on mouse chromosome 2 and evidence of linkage to body fat and insulin on the human homologous region 20q. J Clin Invest 100, 1240-1247

52. Lincoln SE, Lander ES (1992) Systematic detection of errors in genetic linkage data. Genomics 14, 604-610

53. Lincoln SE, Daly M, Lander ES (1992) Constructing genetic maps with MAPMAKER/EXP 3.0, $3^{\text {rd }}$ ed. (Cambridge, Mass.: Whitehead Institute Technical Report)

54. Mackay TF (2001) Quantitative trait loci in Drosophi1a. Nat Rev Genet 2, 11-20

55. Malik RC (1984) Genetic and physiological aspects of growth, body composition and feed efficiency in mice: a review. J Anim Sci 58, 577-590

56. Masinde GL, Li X, Gu W, Davidson H, Ulland MH et al. (2002) Quantitative trait loci (QTL) for lean body mass and body length in MRL/MPJ and SJL/J $\mathrm{F}_{2}$ mice. Funct Integr Genomics 2, 98-104

57. Mehrabian M, Wen P-Z, Fisler J, Davis RC, Lusis AJ (1998) Genetic loci controlling body fat, lipoprotein metabolism, and insulin levels in a multifactorial mouse model. J Clin Invest 101, 2485-2496

58. Melchinger AE, Utz HF, Schon CC (1998) Quantitative trait locus (QTL) mapping using different testers and independent population samples in maize reveals low power of QTL detection and large bias in estimates of QTL effects. Genetics 149, 383-403

59. Moody DE, Pomp D, Nielsen MK, Van Vleck LD (1999) Identification of quantitative trait loci influencing traits related to energy balance in selection and inbred lines of mice. Genetics 152, 699-711

60. Moore KJ, Nagle DL (2000) Complex trait analysis in the mouse: the strengths, the limitations and the promise yet to come. Annu Rev Genet 34, 653-686

61. Morris KH, Ishikawa A, Keightley PD (1999) Quantitative trait loci for growth traits in C57BL/6J $\times$ DBA/2J mice. Mamm Genome 10, 225-228

62. Mullis K, Faloona F, Scharf S, Saiki R, Hom G et al. (1992) Specific enzymatic amplification of DNA in vitro: the Polymerase Chain Reaction. Biotechnology 24, 17-27

63. Nelson MR, Kardia SL, Ferrell RE, Sing CF (2001) A combinatorial partitioning method to identify multilocus genotypic partitions that predict quantitative trait variation. Genome Res 11, 458-470

64. Ott L (1984) An Introduction to Statistical Methods and Data Analysis, $2^{\text {nd }}$ ed. (Boston, Mass.: Duxbury Press)

65. Plum L, Kluge R, Giesen K, Altmuller J, Ortlepp JR et al. (2000) Characterization of a susceptibility locus on chromosome 4 and its relation with obesity. Diabetes 49, 1590-1596

66. Pomp D (1997) Genetic dissection of obesity in polygenic animal models. Behav Genet 27, 285-306

67. Pomp D, Cushman MA, Foster SC, Drudik DK, Fortman $M$ et al. (1994) Identification of quantitative trait loci for body weight and body fat in mice. Proc $5^{\text {th }}$ World Congr Genet Appl Livest Prod 21, 209-212
68. Pomp D, Jerez-Timaure N, Allan MF, Eisen EJ (2002) Integrated genomic, proteomic and metabolomic dissection of polygenic selection response for murine growth and fatness. Proc $7^{\text {th }}$ World Congr Genet Appl Livest Prod 32, 447-450

69. Reifsnyder PC, Churchill G, Leiter EH (2000) Maternal environment and genotype interact to establish diabesity in mice. Genome Res 10, 1568-1578

70. Robertson A (1966) Biochemical polymorphisms in animal improvement. Proc X Europ Congr Anim Blood Groups Biochem Polymorph, 35-42

71. Robertson A (1967) The nature of quantitative genetic variation. In Heritage from Mendel, A. Brink, ed. (Madison, Wis.: University of Wisconsin), pp 265280

72. Rocha JL, Van Vleck LD, Eisen EJ, Pomp D (2004) A large-sample QTL study in mice: II. Organ and body composition traits. Mamm Genome, in press

73. Rocha JL, Siewerdt F, Van Vleck LD, Eisen EJ, Pomp D (2004) A large-sample QTL study in mice: III. Reproduction. Mamm Genome, in press

74. Routman EJ, Cheverud JM (1997) Gene effects on a quantitative trait: two-locus epistatic effects measured at microsatellite markers and at estimated QTL. Evolution 51, 1654-1662

75. SAS Institute Inc. (1985) SAS User's Guide: Basics (Gary, NC: SAS)

76. SAS Institute Inc. (1996) SAS System for Mixed Models (Gary, NC: SAS)

77. Schork NJ (2001) Genome partitioning and whole-genome analysis. Adv Genet 42, 299-322

78. Taylor BA, Tarantino LM, Phillips SJ (1999) Genderinfluenced obesity QTLs identified in a cross involving the KK type II diabetes-prone mouse strain. Mamm Genome 10, 963-968

79. Terwilliger JD, Goring HH (2000) Gene mapping in the $20^{\text {th }}$ and $21^{\text {st }}$ centuries: statistical methods, data analysis, and experimental design. Hum Biol 72, 63-132

80. Van Ooijen JW (1992) Accuracy of mapping quantitative trait loci in autogamous species. Theor Appl Genet 84, 803-811

81. Vaughn TT, Pletscher LS, Peripato A, Ellison KK, Adams E et al. (1999) Mapping quantitative trait loci for murine growth: a closer look at genetic architecture. Genet Res Camb 74, 313-322

82. Visscher PM, Haley CS (1996) Detection of putative quantitative trait loci in line crosses under infinitesimal genetic models. Theor Appl Genet 93, 691-702

83. Wade MJ (1992) Sewall Wright: gene interaction and the shifting balance theory. Oxf Surv Evol Biol 8, 33-62

84. Weiss KM (1996) Is there a paradigm shift in genetics? Lessons from the study of human diseases. Mol Phylogenet Evol 5, 259-265

85. West DB, Lefevre JG, York B, Truett GE (1994) Dietary obesity linked to genetic loci on chromosomes 9 and 15 in a polygenic mouse model. J Clin Invest 94, 1410-1416

86. White JM, Eisen EJ, Legates JE (1970) Sex-heterosis interaction, heterosis and reciprocal effects among mice selected for body weight. J Anim Sci 31, 289-295 
87. Xu Y, Jin P, Mellor AL, Warner CM (1994) Identification of the Fed gene at the molecular level: the Q9 MHC class I transgene converts the Ped slow to the Ped fast phenotype. Biol Reprod 51, 695-699

88. Yano M (2001) Genetic and molecular dissection of naturally occurring variation. Curr Opin Plant Biol 4, 130-135

89. York B, Lei K, West DB (1996) Sensitivity to dietary obesity linked to a locus on chromosome 15 in a
$\mathrm{CAST} / \mathrm{Ei} \times \mathrm{C} 57 \mathrm{BL} / 6 \mathrm{~J} \mathrm{~F}_{2}$ intercross. Mamm Genome 7, 677-681

90. Young ND (1999) A cautiously optimistic vision for marker-assisted breeding. Molec Breed 5, 505-510

91. Zeng Z-B (1993) Theoretical basis for separation of multiple linked gene effects in mapping quantitative trait loci. Proc Natl Acad Sci USA 90, 10972-10976

92. Zeng Z-B (1994) Precision mapping of quantitative trait loci. Genetics 136, 1457-1468 